\title{
A method for work modeling at complex systems: towards applying information systems in family health care units
}

\author{
Alessandro Jatobáa, ${ }^{a}$, Paulo Victor R. de Carvalho ${ }^{\mathrm{b}}$ Amauri Marques da Cunha ${ }^{\mathrm{b}}$ \\ ${ }^{a}$ Escola Nacional de Saúde Pública/Fundação Oswaldo Cruz \\ ${ }^{\mathrm{b}}$ Programa de Pós-Graduação em Informática/Universidade Federal do Rio de Janeiro \\ Rio de Janeiro, Brazil
}

\begin{abstract}
Work in organizations requires a minimum level of consensus on the understanding of the practices performed. To adopt technological devices to support the activities in environments where work is complex, characterized by the interdependence among a large number of variables, understanding about how work is done not only takes an even greater importance, but also becomes a more difficult task. Therefore, this study aims to present a method for modeling of work in complex systems, which allows improving the knowledge about the way activities are performed where these activities do not simply happen by performing procedures. Uniting techniques of Cognitive Task Analysis with the concept of Work Process, this work seeks to provide a method capable of providing a detailed and accurate vision of how people perform their tasks, in order to apply information systems for supporting work in organizations.
\end{abstract}

Keywords: Cognitive Systems Engineering, Health Care, Work Process five

\section{Introduction}

For Nemeth et al. [9] , in complex systems such as health, military, civil aviation and others, in order to understand how employees perform their duties, the real work situations must be observed, given the intrinsic difficulty of decision making in these environments and the variability in their recurrent tasks.

For Cunha e Souza [4], the only way to completely identify a work process is through the identification of who's interested on it and what is expected from its execution. Cunha e Souza also introduce the concept of "boundaries" of the work processes activities, which are represented by the inputs and results of each activity. If these elements are not clearly identified, the work process is not fully described.

Thus, it arises the need of describing the work in the most accurate way, since the farther away from reality the work description is to technology develop- ers, the worse the results are produced by technological devices.

The Cognitive Task Analysis (CTA) [4-11] provides techniques for capturing how human mind works, improving the understanding of how people perform their tasks, or rather how participants see how work is done. When systems are very complex, it is not enough to observe their actions and behaviors. It is necessary to find out what operators are thinking while performing these tasks [2].

This paper proposes a method that combines techniques of CTA and the concept of Work Process in order to describe and represent work in complex systems, to make possible the development of IT solutions to support such work. The proposed method was applied in a unit of the Brazilian Family Health Care Program.

Based on observation of the work of health care professionals, detailed process models were obtained, which are able to represent process boundaries, according to the concept of work process by Cunha e

Corresponding author. E-mail: jatoba@fiocruz.br. 
Souza [4]. It were also gathered cognitive artifacts used as inputs or results of the processes activities. With this material it was carried out an analysis of each activity and it was highlighted "regions" in the processes which have greater cognitive demand. These regions were considered the main opportunities for improvement through IT, and they were submitted to cognitive modeling, which resulted in the production of models of perception and interpretation [2].

It is believed that the method proposed in this paper can make design and implementation of IT to support work in complex systems more effective.

\section{Related work}

\subsection{Process Modeling to Iinvestigate Problems in Information Systems}

The services of medical emergencies are characterized by the need for fast response to users, which can be decisive for injuried people to live or die. Typically, these complex systems make use of some technology to support their activities.

Souza et al. [10] present a systematic approach to identify and investigate the causes of common problems in these systems, making use of process modeling techniques.

The quest for agility in serving people who have suffered severe trauma greatly increases the demand for devices to enhance work. In this sense, Souza et al. present a work that seeks to reduce difficulties in processing of telephone demands for emergency care provided by the Government of Rio de Janeiro. The work's main objective is to present ways to reduce the recording time of information from an occurrence in the software to support the activities of prehospital care.

These kinds of service are quite recent in Brazil and therefore suffer a lot of interference of human factors. People not fully accustomed to this kind of work process usually have difficulties in interacting with existing computing devices.

Based on reports of experts, the research team conducted an exploratory field study, interviewing operators of a Medical Central of Regulation in Rio de Janeiro, and built a set of models of work processes performed by the organization. The team also evaluated the information systems used by the teams.
Initially, the research team sought to obtain detailed knowledge about how work is developed in the organization. For this, the approach made use of the Work Process Concept [4]. The authors have made constant use of the concept of memory in order to map the resources used in carrying out activities, and thus identify and describe the inputs used and outputs produced.

\subsection{Naturalistic Decision Making in complex Systems: the Nuclear Plants Case}

Carvalho et. al [10] examine the cognitive process realized by professionals in decision making while working in a complex system (in this case, a nuclear power plant) to find out if this activity occurs in naturalistic or normative way. The authors evaluated whether the experienced operators in performing a given task consider these tasks familiar, responding to them as naturalistic, or if they compare options before selecting the best possible solution.

The authors state that most abnormalities are preceded by work in a normal regime, so it is necessary to understand how people work during normal operation.

The authors use the approach called Naturalistic Decision Making which refers to how people make decisions in complex systems in the real world.

The field of study of the NDM indicates how experienced people, working individually or in groups in dynamic environments, make decisions and take actions whose consequences are significant not only for them but for the organizations they work [7]

According to Carvalho et al., This approach can be used to find out how contextual factors affect the decision-making process, since this takes into account specific situations, ie, it is necessary to know the situation so that you can understand how the decision was made.

The authors' work suggests the use of a conceptual framework for analyzing the activity of the operators, through which the researcher collects data empirically while interacting with people who are being studied. During the study, the authors collected data through observation of daily work, whether through the production of field notes or through the recording of audiovisual material.

Carvalho et al. used the interactive observation as a technique for collecting data about how work was done at the nuclear plants, which allows researchers to track social conditions that influence the outcome of work, such as conflicts, negotiations between 
actors, consensual arrangements etc.

The results of the study of Carvalho et al. indicate that very automated systems have restrictions related to the hardware used in its operation. The main restrictions are indicated:

- Poor human-system interface;

- Lack of information about the status of automatization;

- Conventional alarm systems;

- Comunication systems;

- Procedures.

The study by Carvalho et al. approaches to this work by suggesting a conceptual framework for analyzing the activity and collect data through observation and interaction as a way to understand and describe work in complex systems as closely as possible to people actually carry out their activities.

\section{The Proposed Method}

In this section the proposed method for the analysis and representation of labor in complex systems is demonstrated. The method's purpose is to provide inputs to a business model more consistent and coherent with the objectives of organizations..

\subsection{Improving the Identification of Business Processes through the Analysis of Cognitive Work}

According to Cunha e Souza [4], a work process is described if you can identify who the interested person is and what is expected of the process achievement. Cunha e Souza also introduce the concept of "boundary" of the work process, which are the inputs in the process activities and the results produced by these processes. If these elements are not clearly identified, the work process was not fully described.

Cunha e Souza aim to provide a formal framework to describe the work done in the organization [5]. The process is a set of activities that must be performed to respond to a client, ie., a specific structure of activities located in time and space, with beginning and end, inputs and outputs identified..

A significant factor with regard to the health care sector can be precisely related to the work process. For Cunha e Souza, a work process can be interpreted as a set of related activities, connected through inputs and outputs. The performance of services is not limited to the application of rules, but requires the use of knowledge (many times, specific) with active participation of the user. So, work does not always occur as planned and, sometimes, integration between those who produce and to whom the service is produced is needed [8].

Cognitive Task Analysis (CTA) provides techniques to capture the functioning of the human mind, enhancing the understanding of how people perform their tasks, or rather, as the participants see how work occurs. When tasks are very complex, it is not enough to observe their actions and behaviors. It is necessary to find out what they were thinking while performing these tasks [2].

Nemeth et al. [9] believe that the study of "cognitive artifacts" [6] allows you to learn details about the work for which they were created to support

Thus, given the complexity of work in the Health Care sector(in this case, more specifically, the Family Health Care Strategy in Brazil), the proposed method suggests the union of techniques of Cognitive Task Analysis[2]. and the concept of Work Process [3-4,5] as a way to describe and represent work in complex systems, to make possible the development of IT solutions to support the work in these systems.

Researchers can identify results and customers of the work processes, processes that have more than one type of customer may also have more than one type of result. The entire process is initiated by one of several types of drives.

These elements are used to identify the boundaries of the work process. We can consider a process fully identified when its boundaries are fully described, that is, one can say what are all the kinds of results, all types of customers, all types of drives and all kinds of triggers. If any of these elements has not been identified, the work process is not properly defined 


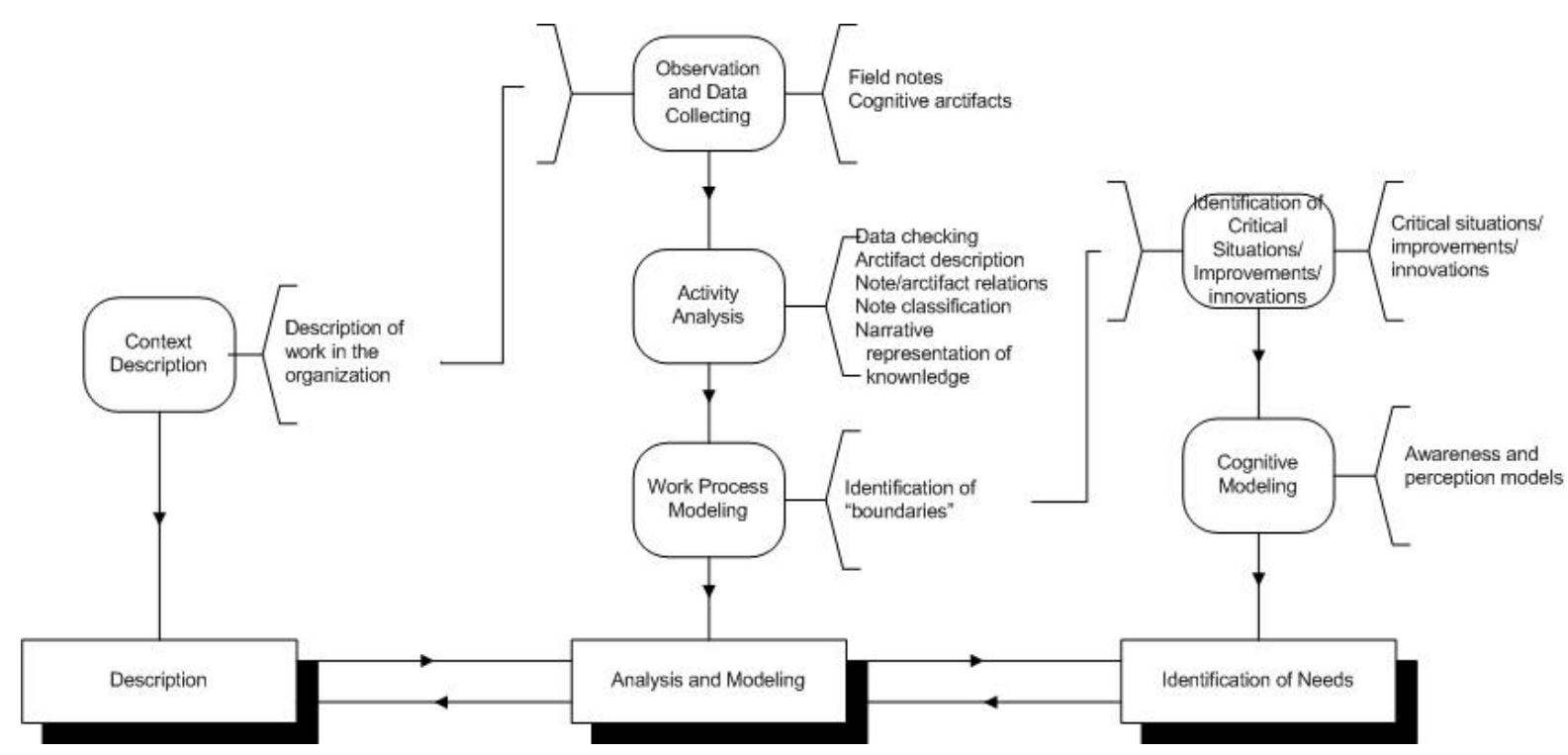

Figure 1 - The Proposed Method

On the other hand, health care environments are characterized by constant decision-making, usually influenced by variables related to the context in which individuals are placed. To make sure these aspects are not ignored in the analysis, it is necessary to describe how people make decisions, assess and act [12].

The proposed method should be used, regardless of the adopted software development process, as a way to improve knowledge about the organization's information system

\subsection{Description of the Proposed Method}

The proposed method has three stages:

- Description

- Context description.

- Analysis and modeling

- Observation and data collecting;

- Activity Analysis;

- Modeling of work processes.

- Identification of needs

- Identification of critical situations, improvements and innovations;

- Cognitive modeling.

Figure 1 illustrates the phases of the proposed method and how its stages relate one to another.

\subsection{Description Stage}

\subsubsection{Context Description}

The objective of Context Description is to obtain knowledge about the organization in which the work is done. It should describe the work environment and provide comprehension about how aspects of the environment influence the work of people.

In this stage, essential features of the work, such as profiles of actors, key working relationships, customer profiles, services provided, division of tasks and organization and team structure must be described. Notes about space and physical structure, its infrastructure and spatial organization should also be taken. More specific features can also be described as the range of services, target audience and working hours.

It is important to identify the actors who should be interviewed, in addition to structure a set of "issues" tab. The questionnaire should be based on issues such as: Who? What? Why? Where? How? What results? When?

At the end of this phase must have been made a preliminary description of the work organization.. 


\subsection{Analysis and Modeling Stage}

The main objective of this stage is to describe how work is developed in the organization. For Nemeth et al [9], in work environments in complex systems, the decision-making is focused on service operator, not the managers. The authors explain that although the cognitive effort of the managers are not less sophisticated, the analysis of operators offer better perspectives on what is developed in the organization.

The method proposed in this work suggests that the analysis and modeling stage must be performed from a set of observations and interactions with the operators. Thus, data on how the work happens in the facility that is the subject of the study can be more efficiently obtained. This approach is suggested by Carvalho et al. as part of a framework for analysis of activity in complex systems [1].

\subsubsection{Observation and Data Collecting}

This phase should be performed by observation the behavior and interactions with those observed. Some conversations and unstructured interviews can be performed, and the collection and assembly of artifacts used in the performance of cognitive tasks can also be taken.

The material must be encoded in field notes. It should also be developed in an iterative manner and the amount of iterations is not defined. The observation notes should contain details of the decisions taken in the course of work, exchange of information between users and workers, relations between professionals and the paths taken in the workplace, as well as, of course, the tasks performed.

\subsubsection{Activity Analysis and Modeling}

Activity Analysis seeks to establish a diagnosis of the operation. Through it researchers can determine what is done, by whom work is done, and the way in which tasks are performed. It should be possible to establish the flows of work, additional methodological steps and interactions [11]

In this phase, CTA concepts are used to elicit cognitive knowledge about how operators perform their tasks, or rather what they think as they do so, and the concept of Work Process [3] is used to identify how the activities relate their.

The concept of Work Process provides a framework to understand and represent the work done by organizations and can also help in deciding how, where and when to adopt technological solutions to support tasks performance.

This aims the formulation of a set of high level models to represent in detail the work done at the organization. A model for each key process can be produced, if the identified models represent variations on a single key process (Cunha and Souza, 2005b)

\subsection{Identification of Needs Stage}

Given the amount of paper used, busy jobs and tasks performed within the organization, it is necessary to make a choice about which cases should undergo intervention and be supported by information systems.

The choice criterion are also opportunities to review the processes, which eventually detail open points and settle disputes. Thus, this choice must be made from a set of criteria, which are indications to define groups of activities that should be more deeply analyzed [11].

In order to provide a way to choose critical situations, the approach suggests a set of the universal criteria of ergonomics. Carvalho and Vidal indicate the following criteria for selection of critical situations:

- Criteria for complaints: Situations in which workers' complaints are many and compelling;

- Criteria for consequences: Cases in which events have the greatest consequence for the professionals;

- Criteria of centrality: Situations that have a relationship with many others in the work of the professionals;

- Criteria for modernity: Situations that require urgent modernization;

- Criteria for stability: The situation that is not, ephemeral and remains throughout the study.

Each key process analysis must form a set of criteria, and from these, point out situations that should be deeply analyzed. The situations chosen are the focus of the next step mostly because they represent the core of work activities

\subsubsection{Cognitive Modeling}

The cognitive modeling can be seen as an activity analysis with specific focus on development of information technology. It assumes that the set of mental activities by operators corresponds to 


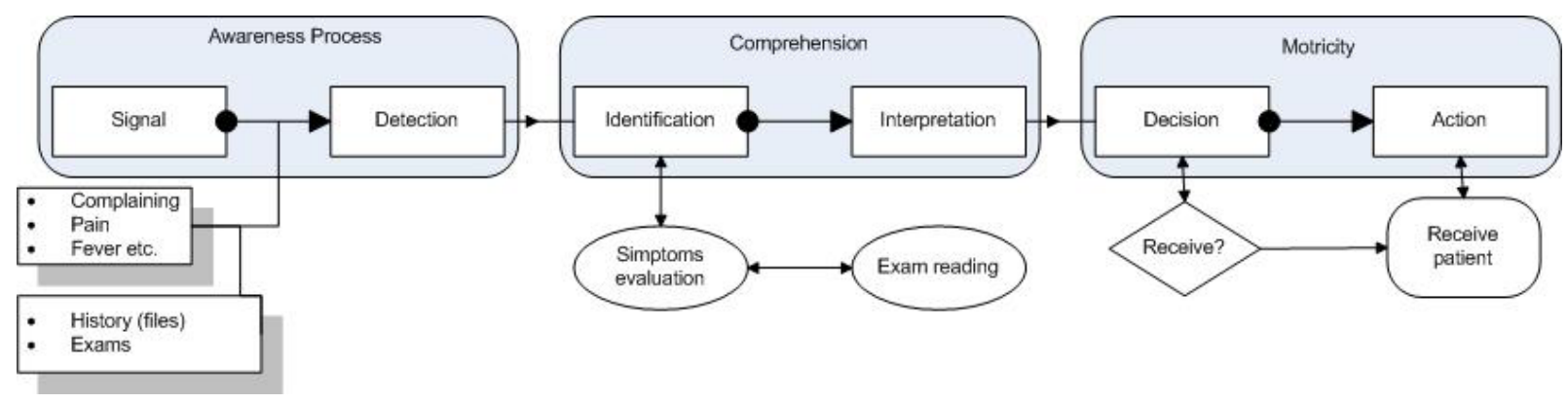

Fig. 2: Awareness and interpretation model

applying a set of skills and behaviors. The basis for cognitive modeling are three types of knowledge (general, operating and routine) as well as the three modes of conduct (skills, rules and knowledge) [11].

In this method, it is suggested the use of models of awareness and interpretation, to focus on the representation of the conditions that allow structural and functional sense, represent and retrieve information used throughout the cognitive process. Given that information systems process information that must be present in the memories of the work process, this type of model is more appropriate.

The memories, long or short, are graded by human beings. According to the model of perception, humans transform information of physical nature in information of symbolic nature, as shown in Figure 2.

Although this type of model brings low formalism and present simple stereotypes, it is able to represent the relationships between mental model building of the operator and the use of memories in this building

At the end of this phase, there should be a set of candidate situations for the adoption of information systems, with their cognition described and illustrated. The models produced during this phase, more than representing the memories, represent how they are used by the operator in performing a certain action, decisions that the operator takes and the memories the can influence those decisions.

\section{Discussion}

The purpose of this paper is to provide a method for modeling work. Compared to traditional modeling techniques, this method should provide greater understanding about how people work, especially in complex systems.

It is also important to ensure that a greater amount of relevant aspects of people's work is present in the models produced, thus ensuring the IT analysts, a wider range of information so that they, together with representatives of the business organization, can have greater ability to decide when to adopt a technological solution.

Thus, at the end of field work, a set of 21 objective questions was presented to professionals of the five teams that were the subject of field work. It was also requested that the unit managers answer the questions.

Those interviews aimed to capture information about how effective the proposed method could be from the point of view of the operators, ie, if the models produced accurately represent the way professionals work.

Questions about the information systems currently used by the organization were also included. It was then possible to verify the compliance of existing systems.

The interviews indicated that the models represent very or completely the work of professionals. Only professionals who do not work in the key process said that there was significant parts of their work not represented in the models.

Importantly, the work focused on key business process. It is therefore natural that sub-unit management processes are not represented in the models.

Although all professionals have stated that the models are complete, almost $20 \%$ claim that the activities are very adequately represented. There were no claims that the work is poorly or inadequately represented. The same goes for questions about the representation of sequences of activities

Regarding the identification of inputs and outputs of activities, including cognitive artifacts, more than half of the interviewed people point to "medium represented". This may indicate little understanding about the products of the activities. In interactions with professionals during the observation, we found 
that many of them have little clarity about the results of its activities with regard to the storage of relevant information. Even when presented to the models, professionals are unclear about whether a particular result is actually produced or not.

This suggests that the proposed method serves its purpose as regards the description of work with a greater degree of reliability, as well as to identify situations where the business is subjected to a greater degree of cognitive effort (intellectual, decision making, pressure etc..) and in the case of the Family Health Strategy, even physical). All these factors have a significant impact in work in complex systems such as public health.

There was also consensus on the fact that the use of software could support the development of subcognitive processes.

This makes it possible to assert that the proposed method makes it possible for professionals to do their reflections on ways of working and thus are able to suggest and implement improvements, whether through IT or not

\section{Conclusions}

The adoption of information systems definitely transforms the ways people work. Although the initiatives of IT in organizations have invariably intended to improve the work, this does not always happen in fact.

To be able to support the work at any level, from management to operational, it is necessary to know how work is developed. Ignorance about how work actually occurs, will inevitably lead to problems in the implementation of assistive devices, either during the development of this, or else during their use.

These problems may be resistance to deployment of the systems, disuse because of poor compliance, or even damage to work because of their poor adaptation to the activities of people.

In some cases, work in organizations is characterized by a huge set of variables. These variables are typically related, ie, a change in one aspect of the job starts a cascading set of variations.

These complex systems add several factors to the work of operators, such as unpredictability, variability and constant decision making. In these systems, the work does not always go as planned, and its operators are obliged constantly to make use of improvisation and are making decisions about how certain activities are performed all the time.
Adopt devices to support operators in these cases becomes even more difficult. Understanding the work in complex systems is an even greater challenge, given that people's work is influenced by a larger set of factors. In these systems, it is necessary to understand not only what operators do, but what goes on inside their minds as they perform their tasks.

This is the contribution of this work, that provide a method for understanding the work of operators in complex systems, taking into account the cognitive effort made by these operators in performing their activities

Through the realization of field work in an environment in public health, specifically in Family Health Care, this paper suggests a way to describe, represent and analyze the work of operators in complex systems, using the concept of Work Process [3-4,5] allied to techniques of Cognitive Task Analysis [2]

With this proposal, which is part of the specification of information systems, more precisely during the modeling business, we believe it is possible to minimize the problems faced in building information systems to support work. During the specification, analysts have the opportunity to get closer to its future users and learn how they perform their activities.

Therefore, since the work, inevitably, is held by persons in complex systems such as public health activities require a huge mental effort, either by the nature of human contact or even through any existing support. It is important, then, that the information systems used must be capable of reducing, or at least not increase the cognitive load of work, besides, of course, they are increasingly tailored to the practices performed.

\section{References}

[1] P.V Carvalho and M.C. Vidal and I.L dos Santos, Nuclear power plant shift supervisor's decision making during microincidents, in: International Journal of Industrial Ergonomics, v.35, n.7, 2005, pp. 619-644.

[2] B. Crandall and G. Klein and R. Hoffman, Working Minds: a practitioner's guide to cognitive task analysis, The MIT Press, 2006.

[3] A. M. Cunha and P. M. da Costa, Towards Key Business Process for E-Government, in: Proceedings of the International Conference on E-Commerce, E-business and Egovernment, 2004.

[4] A.M. Cunha and G. Q. de Souza, Conceito de Processo de Trabalho para Alinhar Sistemas de Informação com os 
Objetivos das Organizações, in: Proceedings of the II Simpósio Brasileiro de Sistemas de Informação, 2005

[5] A.M Cunha and G.Q de Souza Especificando Requisitos a Partir do Conceito de Processo de Trabalho, in: Proceedings of the VII Simpósio Internacinoal de Melhoria em Processos de Software, 2005b

[6] E. Hutchins, Cognition in the Wild. Cambridge, Massachusetts: MIT Press,2002.

[7] G. Klein, Sources of Power. Cambridge, Massachusetts: MIT Press, 2000.

[8] I. Koster, A Gestão do Trabalho e o Contexto da Flexibilização no Sistema Único de Saúde; M.Sc Dissertation, Escola Nacional de Saúde Pública, 2008.
[9] C. Nemeth and M. O'Connor; P. A. Klock and R. Cook, Discovering healthcare cognition: the use of cognitive artifacts to reveal cognitive work, in: Organization Studies,v.27, pp. 1011-1035, 2006

[10]A. P. Souza and J. V. Freitas and A. M. Cunha and M. L. M. Campos;Uma Abordagem Sistemática de Modelagem de Processos para a Investigação de Problemas em Sistemas de Informação: aplicação em uma central de regulação médica., in: Revista Brasileira de Sistemas de Informaçãopp.16-32, 2009.

[11]M. C. Vidal and P. V. de Carvalho, Ergonomia Cognitiva: raciocínio e decisão no trabalho, FAPERJ, 2008.

[12]D. Woods. and E. Hollnagel, Joint Cognitive Systems. Boca Raton, FL,USA: CRC Press, Inc., 2006 